\title{
A close relationship between verbal memory and SN/VTA integrity in young and older adults
}

\author{
Sandra Düzel ${ }^{a}$, Hartmut Schütze ${ }^{\mathrm{e}}$, Sabine Stallforth ${ }^{\mathrm{a}}$, Jörn Kaufmann ${ }^{\mathrm{a}}$, Nils Bodammer ${ }^{\mathrm{a}}$, \\ Nico Bunzeck ${ }^{b}$, Thomas F. Münte ${ }^{c}$, Ulman Lindenberger ${ }^{\mathrm{d}}$, \\ Hans-Jochen Heinze ${ }^{\mathrm{a}}$, Emrah Düzel ${ }^{\mathrm{b}, \mathrm{e}, *}$ \\ a Department of Neurology II and Centre for Advanced Imaging, Otto von Guericke Universität, Leipziger Str. 44, 39120 Magdeburg, Germany \\ ${ }^{\mathrm{b}}$ Institute of Cognitive Neuroscience and Department of Psychology, University College London, 17 Queen Square, London WC1N 3AR, UK \\ ${ }^{\mathrm{c}}$ Department of Psychology II, Neuropsychology Unit, Otto von Guericke Universität Magdeburg, Germany \\ d Center for Lifespan Psychology, Max Planck Institute for Human Development, Lentzeallee 94, D-14195 Berlin, Germany \\ e Institute for Cognitive Neurology and Dementia Research, Otto von Guericke Universität, Leipziger Str. 44, 39120 Magdeburg, Germany
}

\section{A R T I C L E I N F O}

\section{Article history:}

Received 24 January 2008

Received in revised form 2 June 2008

Accepted 8 June 2008

Available online 12 June 2008

\section{Keywords:}

Substantia nigra/VTA

Hippocampus

White matter integrity

Diffusion

Anisotropy

Verbal memory

CVLT

\begin{abstract}
A B S T R A C T
Age-related dysfunction in dopaminergic neuromodulation is assumed to contribute to age-associated memory impairment. However, to date there are no in vivo data on how structural parameters of the substantia nigra/ventral tegmental area (SN/VTA), the main origin of dopaminergic projections, relate to memory performance in healthy young and older adults. We investigated this relationship in a crosssectional study including data from the hippocampus and frontal white matter (FWM) and also assessing working memory span and attention. In groups of young and older adults matched for the variance of their age distribution, gender and body mass index, we observed a robust positive correlation between Magnetization Transfer Ratio (MTR) - a measure of structural integrity - of the SN/VTA and FWM with verbal learning and memory performance among older adults, while there was a negative correlation in the young. Two additional imaging parameters, anisotropy of diffusion and diffusion coefficient, suggested that in older adults FWM changes reflected vascular pathology while SN/VTA changes pointed towards neuronal loss and loss of water content. The negative correlation in the young possibly reflected maturational changes. Multiple regression analyses indicated that in both young and older adults, SN/VTA MTR explained more variance of verbal learning and memory than FWM MTR or hippocampal MTR, and contributed less to explaining variance of working memory span. Together these findings indicate that structural integrity in the SN/VTA has a relatively selective impact on verbal learning and memory and undergoes specific changes from young adulthood to older age that qualitatively differ from changes in the FWM and hippocampus.
\end{abstract}

(c) 2008 Elsevier Ltd. All rights reserved.

\section{Introduction}

Age-related decline in learning and memory, often termed ageassociated memory impairment (AAMI) (Crook et al., 1986), is a well-documented finding in healthy old adults (Balota, Dolan, \& Duchek, 2000; Craik, 1994; Grady \& Craik, 2000; Salthouse, 2003) but the neurobiological underpinnings of this decline are still under debate. A consistent pattern of AAMI is a decrement in episodic memory (Tulving, 1985) apparent in impaired free recall and rec-

\footnotetext{
* Corresponding author at: Institute of Cognitive Neuroscience and Department of Psychology, University College London, 17 Queen Square, London WC1N 3AR, UK. Tel.: +44 207679 4640; fax: +44 2079168517.

E-mail address: e.duzel@ucl.ac.uk (E. Düzel).
}

ollection (Buckner, 2004; Craik, 2006; Hedden \& Gabrieli, 2004; Nilsson, 2003). Evidence from lesion studies in humans and animals indicate that episodic memory is critically dependent on the integrity of the medial temporal lobes (MTL) (Mishkin, Suzuki, Gadian, \& Vargha-Khadem, 1997; Squire, Stark, \& Clark, 2004) and the prefrontal cortex (Stuss \& Levine, 2002). Therefore, recent studies have sought to investigate the relationship between structural age-related degeneration of grey and white matter in these regions and learning and recall (Brickman, Habeck, Zarahn, Flynn, \& Stern, 2007; Buckner, 2004; Craik, 2006; Mungas et al., 2005; Schiltz et al., 2006). However, it has also been pointed out that AAMI is not only a result of degeneration of prefrontal and medial temporal brain regions, but also a result of age-related dysfunction in their dopaminergic (DA) (Backman, Nyberg, Lindenberger, Li, \& Farde, 2006) neuromodulation, a finding that can potentially motivate 
new pharmacological treatment strategies for age-related memory dysfunction. The focus of the present study is on these neuromodulatory influences.

Investigations into the relationship between dopamine and AAMI have been fuelled recently by improved understanding of the role that dopamine plays in memory formation (Lisman \& Otmakhova, 2001) and the age-related degeneration of dopaminergic circuitry (Backman et al., 2000; Backman et al., 2006). There is converging evidence that dopamine is not only critically involved in reinforcement learning but also in hippocampus-dependent episodic memory formation (Lisman \& Grace, 2005). Functional anatomical evidence for the role of dopaminergic midbrain in episodic encoding comes from recent studies using functional magnetic resonance imaging (fMRI). Reward-related activation of the substantia nigra/ventral tegmental area (SN/VTA), the origin of dopaminergic neuromodulation, is associated with improved hippocampus-dependent long-term memory formation and possibly consolidation (Adcock, Thangavel, Whitfield-Gabrieli, Knutson, \& Gabrieli, 2006; Wittmann et al., 2005). Encoding-related midbrain activation also occurs independently of reward (Schott et al., 2006). Indeed, as in animals (Lisman \& Grace, 2005), also the human SN/VTA responds to stimulus-novelty in the absence of reward (Bunzeck \& Duzel, 2006). These data provide evidence in favour of a recent model suggesting a functional hippocampal-SN/VTA loop of novelty-processing and encoding (Lisman \& Grace, 2005). Furthermore, dopamine plays a critical role in regulating the subcortical flow of information between medial temporal structures such as the hippocampus and prefrontal cortex (Grace, Floresco, Goto, \& Lodge, 2007). Hence, dopaminergic dysfunction in aging should impact on novelty processing, long-term consolidation as well as on learning tasks that depend on the functional interplay between prefrontal and medial temporal brain regions.

What makes this functional relationship between SN/VTA activity and memory formation particularly relevant for aging research is that dopaminergic neuromodulation undergoes age-related degeneration (for a review see Backman et al., 2006). Human autopsy data indicate a 3\% age-related decrease in dopamine D1 (Cortes, Gueye, Pazos, Probst, \& Palacios, 1989; Rinne, Lonnberg, \& Marjamaki, 1990; Seeman et al., 1987) and D2 receptors (Seeman et al., 1987) per decade. There is a 2-6\% loss of dopaminergic neurons in the SN per decade (Fearnley \& Lees, 1991a) and this loss is correlated with the decrease in striatal dopamine availability (Snow et al., 1993). In older adults, behavioural deficits in episodic memory are better accounted for by D2 receptor binding than by age (Backman et al., 2000). Recently, Bunzeck et al. (2007) quantified age-related structural degeneration of the mesolimbic system in healthy elderly using magnetization transfer ratio (MTR) and correlated it with mesolimbic hemodynamic responses (HRs) to stimulus-novelty. Their findings support the model of a hippocampal-SN/VTA loop of mesolimbic novelty processing by showing that the hemodynamic activation in SN/VTA and hippocampus for novelty is selectively affected by age-related degeneration of these structures.

Magnetization transfer in tissue relates to the exchange of proton magnetization between mobile water protons and protons that are immobilized by macromolecules (Wolff \& Balaban, 1989). MTR reductions have been observed in the SN in patients with Parkinson's disease (PD) (Eckert et al., 2004; Seppi \& Schocke, 2005). PD is characterized by a selective depletion of dopaminergic, neuromelanin-containing neurones of the $\mathrm{SN}$ (pars compacta). Neuromelanin is the dark insoluble macromolecule that confers the black colour to the SN. Neuronal loss as well as degradation of the neuromelanin macromolecule scaffolding (Fasano, Bergamasco, \& Lopiano, 2006) could lead to a reduction of MTR and it is conceivable that both mechanisms could also lead to some reduction in MTR in apparently healthy older adults who do not have PD. Together with our recent observation that SN/VTA MTR is correlated with the magnitude of hemodynamic novelty responses in the mesolimbic system (Bunzeck et al., 2007), these properties make MTR an interesting imaging modality for studying the relationship between structural SN/VTA integrity and memory in aging.

In the present study we investigated whether degeneration of components of the mesolimbic system (SN/VTA and hippocampus) as quantified by MTR is related to age-associated decline in list learning and recall. To that end, we matched a group of 21 older adults (mean age of 65 years) and 21 younger adults with respect to the variance of their age distribution, their gender, and, to minimize differences in health status also body mass index (Table 1). Given that SN/VTA and the hippocampus although being grey matter structures also contain white matter, we used frontal white matter as a 'reference' region for the structural integrity of white matter tracts. We hypothesized that integrity of the SN/VTA is correlated positively with verbal learning because dopaminergic dysfunction should impact on novelty processing - an early stage of declarative long-term memory encoding (Tulving, Markowitsch, Craik, Habib, \& Houle, 1996) - as well as the functional interplay between prefrontal and medial temporal brain regions. A widely used example of a verbal learning and memory task, the California verbal Learning and Memory task (Delis, Kramer, Kaplan, \& Ober, 1987), was used here. Alternatively, degeneration in this region might have a less selective impact and might also impair working memory and attention. The issue of specificity is of general importance as it is still unclear to what extent certain neurobiological changes in aging specifically influence learning and memory in comparison to other cognitive faculties that also show age-related decline and have been shown to be correlated with learning and memory performance in aging, in particular measures of executive functions (Kray \& Lindenberger, 2000; Lindenberger, Marsiske, \& Baltes, 2000; Parkin \& Java, 1999), working memory (Baddeley, Cocchini, Della Sala, Logie, \& Spinnler, 1999) and processing speed (Salthouse, 2000).

\section{Methods}

\subsection{Participants}

86 older adults aged above 55 (mean age $=65$ years, age range $=55-82$ years, S.D. $=5.6$ years, 36 males) and 21 young adults aged between 18 and 30 (mean age $=23$ years, age range $=21-30$ years, S.D. $=2.2$ years, 8 males) participated in the study. All participants were native speakers of German. Exclusion criteria for both groups were a history of neurological and psychiatric disorders, cerebral vascular disease, drug addiction, metabolic diseases like diabetes mellitus, metallic implants, tinnitus, obesity, a Geriatric Depression Scale with a depression score of more the five points (GDS ranges from 0 to 15; scores of higher than 11 indicate depression), a Mini-Mental State lower than 27 (MMSE ranges from 0 to 30; scores of lower than 25 are taken as indicators of pathology), and severe untreated hypertonia. Individuals with mild hypertonia according to the World Health Organization (WHO) and International Society of Hypertonia (ISH) classification (WHO \& ISH, 1999) of hypertonia who were treated with one antihypertensive medication were eligible for participation in the study. The Local Ethics Committee of the University of Magdeburg (Germany) approved the study. All participants gave written informed consent.

\subsection{Procedure}

Participants were recruited using local newspaper announcements. Initial screening for exclusion or inclusion criteria was done using a structured phone interview with around 120 volunteers. 100 eligible volunteers were invited for neuropsychological and psychosocial assessment. After neuropsychological testing (duration of $90 \mathrm{~min}$ ) participants completed psychosocial interviews and questionnaires (duration of $40 \mathrm{~min}$ ) the results of which will be reported elsewhere. Immediately after neuropsychological and psychosocial testing all participants underwent Doppler-sonography of the extra- and intracranial vessels by a trained neurologist. Blood pressure was measured bilaterally, and a blood sample was taken for genetic studies (results are not reported here). If none of the exclusion criteria was met and audition and corrected vision was sufficient to allow neuropsychological testing, MR imaging was conducted within a week after neuropsychological and psychosocial assessment. After inspecting proton-density and T2 weighted MR 
Table 1

Neuropsychological test scores and regional structural parameters of old $(N=21)$ and young $(N=21)$ adults

\begin{tabular}{|c|c|c|c|c|c|}
\hline & \multicolumn{2}{|c|}{ Younger adults } & \multicolumn{2}{|c|}{ Older adults } & \multirow[t]{2}{*}{$p$-Value } \\
\hline & Mean & S.D. & Mean & S.D. & \\
\hline AGE & 23.42 & 2.25 & 64.24 & 2.25 & \\
\hline Body mass index & 23.01 & 3.6 & 23.9 & 3.22 & .398 \\
\hline CVLT total & 65.45 & 7.7 & 55.62 & 9.77 & $.001^{*}$ \\
\hline CVLT delayed recall & 14.5 & 1.97 & 11.9 & 2.95 & $.002^{*}$ \\
\hline Digit span & 6.67 & 1.11 & 6.24 & 1.13 & .224 \\
\hline $\mathrm{D} 2$ & 507.38 & 76.57 & 354.33 & 77.42 & $.000^{*}$ \\
\hline MTR SN right & .467 & .009 & .467 & .009 & .900 \\
\hline ADC SN right & 849.58 & 51.58 & 798.05 & 42.02 & $.001^{*}$ \\
\hline AI SN right & 392.36 & 43.78 & 337.89 & 38.84 & $.000^{*}$ \\
\hline MTR Hippocampus right & .441 & .011 & .441 & .015 & .921 \\
\hline ADC Hippocampus right & 871.10 & 30.6 & 887.11 & 54.15 & .245 \\
\hline AI Hippocampus right & 115.54 & 13.84 & 115.19 & 29.88 & .962 \\
\hline MTR frontal white matter right & .526 & .009 & .52 & .01 & .025 \\
\hline AI frontal white matter right & 288.81 & 22.81 & 268.19 & 27.69 & .012 \\
\hline ADC frontal white matter right & 773.24 & 20.54 & 780.95 & 43.38 & .466 \\
\hline
\end{tabular}

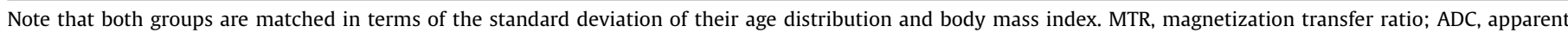

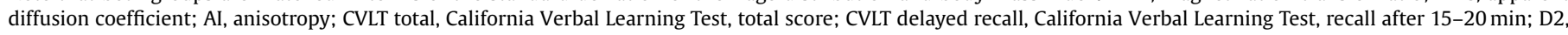
total score in the D2 test of attention.

Significant group differences, see text for procedures correcting for multiple comparisons.

images and excluding individuals with MR vascular lesions, 86 older adults aged above 55 (mean age $=65$ years, age range $=55-82$ years, S.D. $=5.6$ years, 36 males) formed the pool of older adults. Young participants were students or employees of Magdeburg University and contacted and selected according to the same criteria as the older adults.

\subsection{Matching between young and older adult groups}

The goal of the present investigation was to assess the age-related changes in the relationship between structural integrity in the mesolimbic system (SN/VTA and hippocampus) using frontal white matter as a 'reference' region for the structural integrity of white matter tracts. To that end, we matched a group of 21 older adults (mean age of 65 years) to our 21 younger adults with respect to the variance of their age distribution (Table 1 ). We also ensured that the two groups were exactly matched in terms of gender-distribution ( 8 males and 13 females in either group) and did not differ in terms of their body mass index (Table 1 ). The latter was to minimize differences in health status between the groups. Of the 21 older adults, only two had mild hypertonia (140-159/90-99 mmHG) and both were treated with an ACE-Inhibitor.

\subsection{Neuropsychological testing}

The neuropsychological test battery was administered to each participant to provide an estimate of cognitive functioning including measures of learning and memory performance, attention/processing speed, working memory, verbal fluency and general knowledge. The following neuropsychological tests were administered to all participants: The 'California verbal learning Test' (CVLT) (Delis et al., 1987); the 'Diagnosticum für Cerebralschädigung' (DCS) (Weidlich \& Lamberti, 1993); the Digit Span test (digit span forward; digit span backwards); a word fluency assessment (adapted German version of the 'Controlled Oral Word Association Test' (COWAT) (Ruff, Light, Parker, \& Levin, 1997); the 'Trail Making Test' part A and B (Reitan, 1992); the 'd2 test of Attention' and the 'Mehrfachwahl-Wortschatztest' (MWT B, an estimate of general knowledge or 'crystallized intelligence') (Lehrl, 2005). The Mini mental status examination (MMSE) and the Geriatric depression scale (GDS) were administered to older adults only. For the purpose of the current report, results only from the CVLT, Digit Span forward and the D2 test of Attention will be reported.

The CVLT is a verbal list learning and recognition and recall test. It measures the ability to learn by repetition and also taps into recognition and episodic memory (delayed free recall and cued recall). A list of 16 semantically related shopping items (List A) is verbally presented in five trials (Trial 1-Trial 5). The participant is asked to recall as many items as possible after each list is verbally presented. Total free recall for trial 1 (T1) assessed initial recall and for trial 5 (T5) assessed recall after five repetitions. To assess interference effects an additional 16-item shopping list (List B) is administered once after the fifth learning trial. Free and cued recall is assessed immediately after presentation of the interference list (Trial 7 ) as well as after a 15-20-min delay (delayed recall). Total recall summed across Trials 1 through 5 (T1-T5) provided a measure of learning and memory performance (henceforth termed CVLT total, a higher score means better memory performance)

\subsection{Image acquisition}

For both the young and older adults a comprehensive structural data set was acquired in one MRI session on a GE Medical Systems Signa 1.5 T MRI scanner.

\subsubsection{T1-weighted anatomical images}

For each subject a T1-weighted sagittal 3D scan (contrast-optimized spoiled gradient-echo sequence, 124 slices, $256 \times 256$ pixel matrix, field of view $(\mathrm{FOV})=250 \mathrm{~mm} \times 250 \mathrm{~mm}$, slice thickness $=1.5 \mathrm{~mm}, \mathrm{TE}=8 \mathrm{~ms}, \mathrm{TR}=24 \mathrm{~ms}$; flip angle $=30^{\circ}$, leading to a voxel size of $0.98 \mathrm{~mm} \times 0.98 \mathrm{~mm} \times 1.5 \mathrm{~mm}$ ) was acquired These images were later used for further analyses using optimized automatic segmentation procedures (Good et al., 2001).

\subsubsection{Combined proton density (PD)/T2 measurements}

A series of intermitting T2-weighted and proton-density-(PD)-weighted transversal slices was acquired for each subject and subsequently separated into one T2-volume and one PD-volume. Each volume had a resolution of $256 \times 256$ pixels in 44 slices, voxel size $=0.97 \mathrm{~mm} \times 0.97 \mathrm{~mm} \times 3.0 \mathrm{~mm}$. These images were used to identify possible lesions from strokes or other brain diseases and for anatomica localization.

\subsubsection{Diffusion tensor imaging}

Diffusion-weighted images were achieved using a single-shot diffusionweighted spin-echo-refocused EPI sequence $(256 \times 256$ interpolated pixel matrix $\mathrm{FOV}=280 \mathrm{~mm} \times 280 \mathrm{~mm} ; \mathrm{TE}=70 \mathrm{~ms} ; \mathrm{TR}=10,000 \mathrm{~ms} ; 39$ slices; slice thickness $=3 \mathrm{~mm} ; b$-value $=1000 \mathrm{~s} / \mathrm{mm}^{2}$ ), acquired in 12 non-collinear gradient orientations, each measured with the opposite diffusion gradient polarity. The orientations were chosen according to the DTI acquisition scheme proposed by Papadakis et al. (1999). The total of 24 diffusion-weighted measurements, each an average of four measurements, were divided into four blocks, each preceded by a non-diffusion-weighted acquisition. The DTI images were Eddy-current-corrected according to the correction scheme developed by Bodammer, Kaufmann, Kanowski, and Tempelmann (2004), followed by a correction for head motion on the basis of the non-diffusion-weighted images using the AIR software package (Woods, Grafton, Holmes, Cherry, \& Mazziotta, 1998). Diffusion tensors were calculated for each voxel by singular value decomposition and then decomposed into eigenvalues and eigenvectors. Based on the eigenvalues the apparent diffusion coefficient (ADC) and fractional anisotropy (FA) were computed. This post processing resulted in transversal ADC-, FA-volumes with a voxel size of $1.09 \mathrm{~mm} \times 1.09 \mathrm{~mm} \times 3.0 \mathrm{~mm}$.

\subsubsection{Magnetization transfer imaging}

To achieve MT imaging, the magnetization of macromolecular protons is partially saturated using appropriate off-resonance irradiation during standard proton density weighted imaging. The interaction of these partially saturated macromolecular protons with the protons of mobile water in their direct surrounding attenuates the observed water signal in the images. This signal reduction depends on tissue properties such as the concentration, structure and/or chemistry of macromolecules and water content as well as on image sequence parameters.

MT imaging was performed using two volumes with identical settings (transversal, $256 \times 256$ pixels, 48 slices, TE/TR $20 / 2600 \mathrm{~ms}$ ) voxel size $(0.98 \mathrm{~mm} \times 0.98 \mathrm{~mm} \times 3.0 \mathrm{~mm})$. The first one $(\mathrm{MT})$ with a saturation pulse $(1200 \mathrm{~Hz}$ 


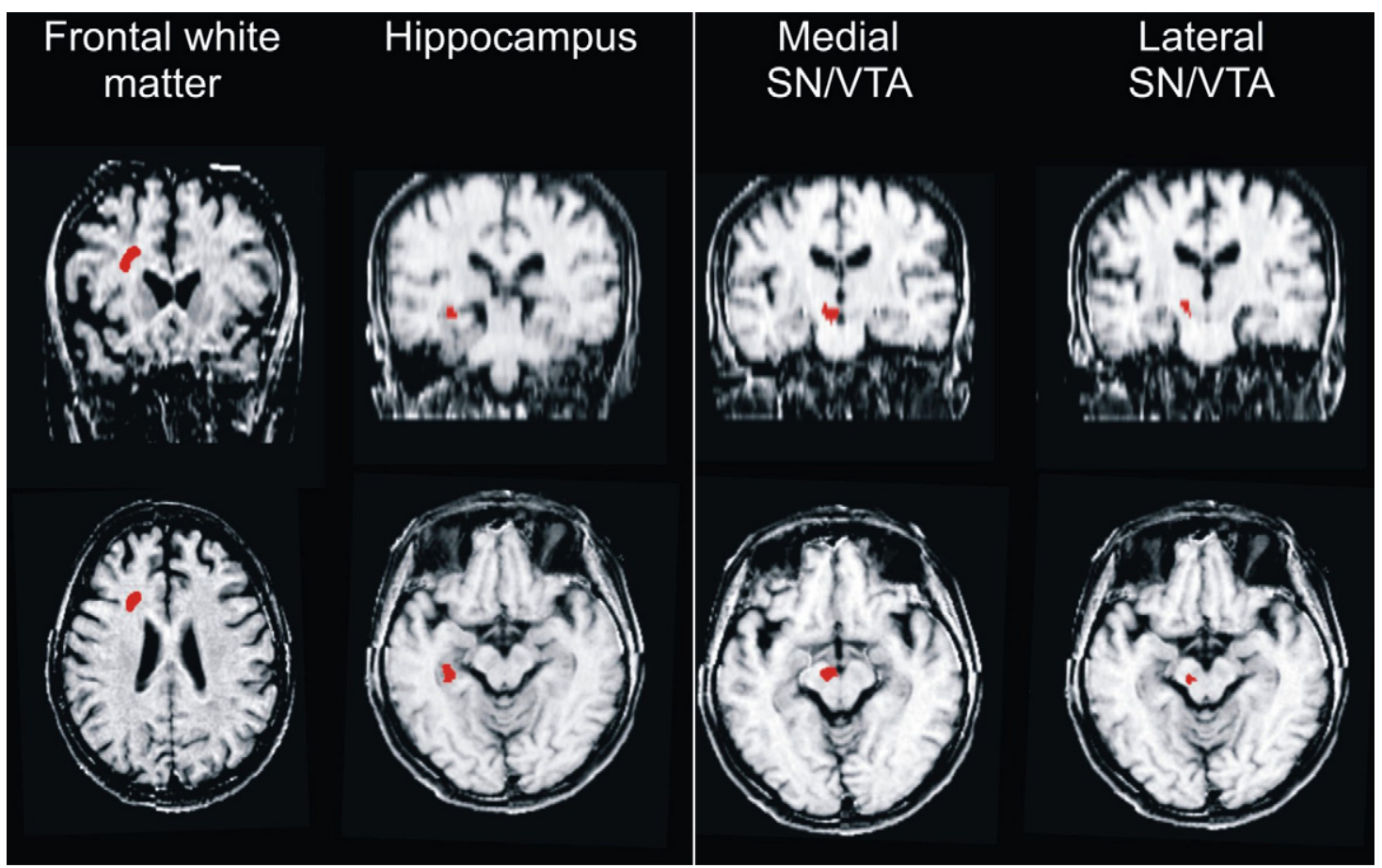

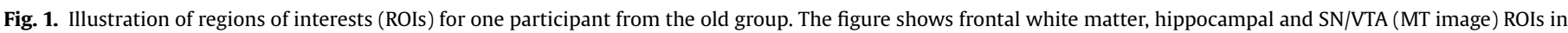
a coronal and transversal view.

off-resonance, $16 \mathrm{~ms}$ ) and the second one without (noMT) a magnetic saturation pulse resulting in a PD-like image. Subsequently, the magnetization transfer ratio (MTR) maps for each subject were calculated on a voxel by voxel basis according to: MTR $=($ noMT-MT $) /$ noMT

\subsection{Regions of interests (ROIs)}

Regions of interests were defined as follows (Fig. 1). From each ROI, mean MTR, $\mathrm{ADC}$ and $\mathrm{AI}$ measures were extracted.

\subsubsection{Substantia nigra/VTA ROI (SN-ROI)}

All boundaries of the substantia nigra/VTA were selected visually based on the intense contrast change between its bright grey colour and the dark grey colour of the adjacent tissue in the MT-image. First the SN/VTA-ROI was defined as a whole and then later was divided into a medial (mSN/VTA-ROI) and lateral part (ISN/VTAROI) (Fearnley \& Lees, 1991b). The upper limit of the SN/VTA-ROI was selected at a level of the superior colliculi where the cross sectional area of the SN/VTA appeared as an even bright grey coloured area in the MT-image, hence excluding voxels that directly flank the adjacent tissue. The anterior part of the SN/VTA-ROI was limited by the interpeduncular fossa and posterior borders were limited by the lateral side of the cerebral peduncle. The medial and lateral boundaries of the SN/VTA-RO were extended until the contrast changed. The lower limit of the SN/VTA-ROI was identified as the last even grey coloured cross sectional area. The total rostrocauda extension of the ROI ranged between 3 and 4 axial slices $(9-12 \mathrm{~mm}$ ) depending on the individual size of the substantia nigra/VTA. According to the study of Fearnley and Lees (1991b), the medial and lateral parts of the SN/VTA-ROI were separated by deleting a diagonal line of voxels within the SN/VTA-ROI. The junctures of the diagonal were defined by the midpoint of the ventral side of the cerebral peduncle and its intersection with an imaginary line connecting the anterior and posterior intersection of the superior sagittal sulcus at an angle of about $45^{\circ}$. Note that Fearnley and Lees (1991b) further subdivided into a ventral and dorsal tier, but the resolution of our MT imaging did not allow for such fine-grained subdivision. For the purpose of the current investigation, data from the lateral and medial SN/VTA portions were collapsed.

\subsubsection{Hippocampal ROI (HC-ROI)}

The upper boundary of the SN-ROI served as a reference for the upper limit of the HC-ROI. The anterolateral boundaries were defined by the extensions of the lateral ventricles. The medial-posterior part of the HC-ROI was limited by the transition into the entorhinal cortex. To exclude any voxels containing cerebrospinal fluid, a safety margin of about two voxels was left between hippocampal tissue and the surrounding structures. The rostro-caudal extension of the HC-ROI comprised 3 slices $(9 \mathrm{~mm})$.
The ROI selection for the hippocampus and SN/VTA ROIs was conducted on individual MR data by S.S. and E.D. Both were blind with respect to age and sex of the participant. Interrater reliability could not be determined here because ROIs were not used for volumetric purposes.

\subsubsection{Frontal white matter (FWM-ROI)}

One common ROI (in each hemisphere) was defined on the average anisotropy image of all participants. The centre of the ROI was placed in the white matter junction of the superior, medial and inferior frontal gyri just anterior of the tip of the third ventricle and lateral to the corpus callosum. The ROI extended $1 \mathrm{~cm}$ in anterior/posterior and superior/inferior directions and around $3 \mathrm{~mm}$ laterally and medially. Care was taken that the ROI did not include any CSF in individual participants. To that end, the location of the ROI was checked on both the normalized AIand ADC images of each participant.

\subsubsection{Co-registration of ROIs from different imaging modalities}

The PD and T2 images were acquired in the same measurement, and are already coregistered. The same applies to the MT and noMT images of the MTratio series, and to the images of the DTI series (meanB0, ADC and AI images). The T2 image was then normalized to the SPM T2 template in MNI space. Since MT, meanB0 and T2 have the same internal contrast, we normalized the individual MT image to the normalized T2 image, masked with a smoothed brain mask that only includes brain tissue, but not muscle, skin or skull. The parameters gained by this normalization were used to warp the noMT images as well as the calculated MTratio images. The meanB0 image of the DTI series was also normalized to the masked and normalized T2 image. The parameters were then applied to the ADC- and AI-maps. On visual inspection, the resulting image sets of individual subjects showed near perfect intra-individual alignment of medial as well as lateral structures, and also diminished the effects of the typical susceptibility artifacts over the frontal sinuses. In a second step, the individual normalized images were used to calculate mean images as templates for each modality, and normalize all individual images a second time to these templates. This was done to get a better inter-individual alignment. As a result, it is possible to directly compare results of group statistics of different modalities. ROIs were defined in the modality (native space) where the structure was most discernable, and the normalization parameters, gained from normalizing the original image to other modalities, were used to warp the ROIs to be in the space of the other modality. Thus we could get mean data for all modalities (i.e. ADC, AI, MTr) from defined ROIs (i.e. SN/VTA, FWM, Hippocampus).

\subsubsection{Statistical analyses}

Statistical analyses were performed using SPSS (Version 11). Planned pairedcomparisons (independent samples $t$-test, two-tailed) between young and older adults were conducted for CVLT total scores, CVLT delayed recall scores, the Digit Span and D2 performance, and all three imaging modalities from all three ROIs. This 
amounted to 14 paired comparisons. The significance threshold after Bonferroni correction was at $p \leq .003$.

Planned correlations between structural and cognitive measures included CVLT total scores, CVLT delayed recall scores, the Digit Span and D2 performance and MTRs from the SN/VTA, the hippocampus and frontal white matter ROIs. As our main goal was to assess the relationship between SN/VTA integrity and cognitive performance, and our previous data (Bunzeck et al., 2007) suggest that MTR is a suitable measure of SN/VTA integrity, the correlations were conducted using MTR values only (to reduce the problem of multiple comparisons). The right SN/VTA region has been consistently implicated in functional imaging studies of hippocampusdependent memory formation using both pictorial (Bunzeck et al., 2007; Wittmann et al., 2005) and verbal (Schott et al., 2006) material. We therefore restrained our analyses to only the right-sided ROIs(however data for the left sided ROIs can be made available from the first author upon request). Given our a priori hypothesis based on our previous work (Bunzeck et al., 2007) that CVLT scores would be correlated with SN/VTA integrity in older adults, these specific correlations were corrected only for 2 comparisons (CVTL total score, CVLT delayed recall). All other remaining correlations between the four cognitive scores and MTRs from the three ROIs in the young and older group (the remaining 22 correlations) were corrected for multiple comparisons using Bonferroni correction (significance threshold $p \leq .002$, two-tailed).

Measures of anisotropy and diffusion were used to interpret the MTR changes rather than detecting additional correlations between cognitive and psychosocial variables and structural integrity. Because we could not formulate strong a priori hypotheses for these comparisons, Bonferroni correction was applied for $12 \mathrm{com}-$ parisons (MTR values from 3 ROIs correlated with two diffusion-related measures (AI and $A D C$ ) in young and older adults) leading to a significance threshold at $p \leq 0.004$ (two-tailed).

The correlation analyses were followed up by multiple regression analyses to determine which combinations of MTR values from the three different regions or combinations of MTR values and diffusion values would best explain observed variance of cognitive variables.

\section{Results}

\subsection{Health status}

None of the older and young participants had a serious health problem, or excessive alcohol or tobacco consumption, while $30 \%$ were current or former smokers. For the older adults the mean BMI was 23.09 (S.D. = 3.22) and for the young adults 23.01 (S.D. 3.6).

\subsection{Cognitive performance}

Compared to the younger group, older participants achieved lower scores on all neuropsychological measures of interest except working memory span (Digit Span) (Table 1).

\subsection{Structural data}

In a first analysis, the mean magnetization transfer ratio (MTR), apparent diffusion coefficient (ADC) and anisotropy (AI) values of the ROIs from SN/VTA, hippocampus and frontal white matter were extracted for all subjects and compared between the matched groups of young and older participants. Independent two-sample $t$-tests between the matched groups showed significant differences between right frontal white matter MTRs (Table 1) with higher
MTR-values for the younger group compared to the group of older subjects. There was no significant difference between both groups' right hippocampal MTRs, and right SN/VTA MTRs (Table 1). None of the structural measures showed a correlation with age (all $p s>.2$ ) except for MTR in the FWM ROI (Fig. 3).

In an earlier publication (Bunzeck et al., 2007) we had reported that SN/VTA MTR was significantly lower in older than in younger adults. Unlike the present study, we had not matched young and old groups in terms of BMI in that earlier study, raising the possibility that BMI - as an indication for general health status and physical activity levels - may be related to age-related decreases in SN/VTA MTR. We assessed this possibility by comparing SN/VTA MTRs of the young adults to those from 12 older adults with the lowest BMI (mean BMI = 21.1, S.D. 1.16) and 12 with the highest BMI (mean BMI $=32.5$, S.D. 2.2 ) values sampled from the entire group of 86 older adults. As can be seen in Fig. 3, SN/VTA MTRs in the high BMI group (mean MTR $=0.458$, S.D. 0.011 ) were significantly lower than those of the young adults (independent samples $t$-test assuming unequal variances, $t=-2.4, p=0.022$, two-tailed) and the older adults with low BMI (mean MTR $=0.468$, SD 0.009; independent samples $t$-test, $t=-2.3, p=0.03$, two-tailed). This relationship to BMI distinguishes the age-related changes in SN/VTA MTR from those in the FWM which, as reported above, were lower in older than younger adults even after matching for BMI.

\subsubsection{Relationship between CVLT performance and regional MTR}

In the group of the young adults MTRs from the right SN/VTA showed a negative correlation with CVLT total (Fig. 2). Even though there was a trend for a negative correlation between the hippocampus MTR and CVLT total, and FWM MTR and CVLT total (Fig. 2) both correlations did not reach statistical significance after Bonferroni correction (Table 2). Partial correlations controlling for age diminished these relationships (Table 2). In the older group, the opposite pattern emerged. Here, the CVLT total scores correlated positively with the right SN/VTA (Fig. 2), and there was a clear trend towards a positive correlation between CVLT total and FWM which again did not reach statistical significance after Bonferroni correction (Fig. 2). There were no further statistically significant correlations between any of the cognitive measures and defined ROIs (Table 2).

\subsubsection{Relationship between regional MTR and diffusion coefficients $(A D C)$ and MTR and anisotropy (FA)}

In order to better understand why correlations between regional MTRs and CVLT total were so different between young and old adults, we assessed the relationship between MTR and ADC and AI from the same individual regions of interest. In the group of the young adults, there were no statistically significant correlations between regional MTR values and ADC or regional MTR values and AI (all ps >.3, Table 3). However, in the older adults, MTR and ADC were negatively correlated in the frontal white matter (Fig. 2, Table 3). AI, however, was not reliably correlated with MTR in either of the regions (Table 3 ). Note that the strong negative correlation

Table 2

Correlation coefficients (Pearson's correlation) for the correlations between regional measures of MTR and test scores for young (upper half) and older (lower half) adults

\begin{tabular}{|c|c|c|c|c|c|c|c|c|}
\hline & \multicolumn{2}{|l|}{ CVLT total } & \multicolumn{2}{|c|}{ CVLT delayed recall } & \multicolumn{2}{|c|}{ Digit Span fwd } & \multicolumn{2}{|l|}{ D2 } \\
\hline & $r$ & $p$-Value & $r$ & $p$-Value & $r$ & $p$-Value & $r$ & $p$-Value \\
\hline SN/VTA right & $-.641^{*},-.594$ & $.002, .007$ & $-.445,-.509$ & $.049, .025$ & .335 & .137 & -.057 & .806 \\
\hline Hippocampus right & $-.509,-.451$ & $.022, .053$ & -.283 & .226 & .150 & .516 & -.260 & .256 \\
\hline Frontal white matter right & $-.519,-.445$ & $.019, .056$ & -.285 & .223 & .242 & .290 & -.039 & .867 \\
\hline SN/VTA right & $.497^{*}, .498^{*}$ & $.022, .025$ & .173 & .454 & .320 & .157 & .342 & .129 \\
\hline Hippocampus right & .218 & .342 & .260 & .254 & .458 & .038 & .283 & .215 \\
\hline Frontal white matter right & $.524, .462$ & $017, .035$ & .327 & .148 & .237 & .300 & .412 & .064 \\
\hline
\end{tabular}

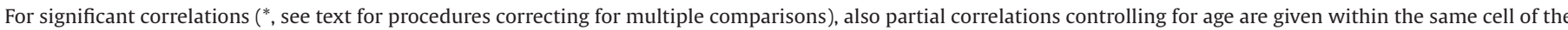
table. 
(A)
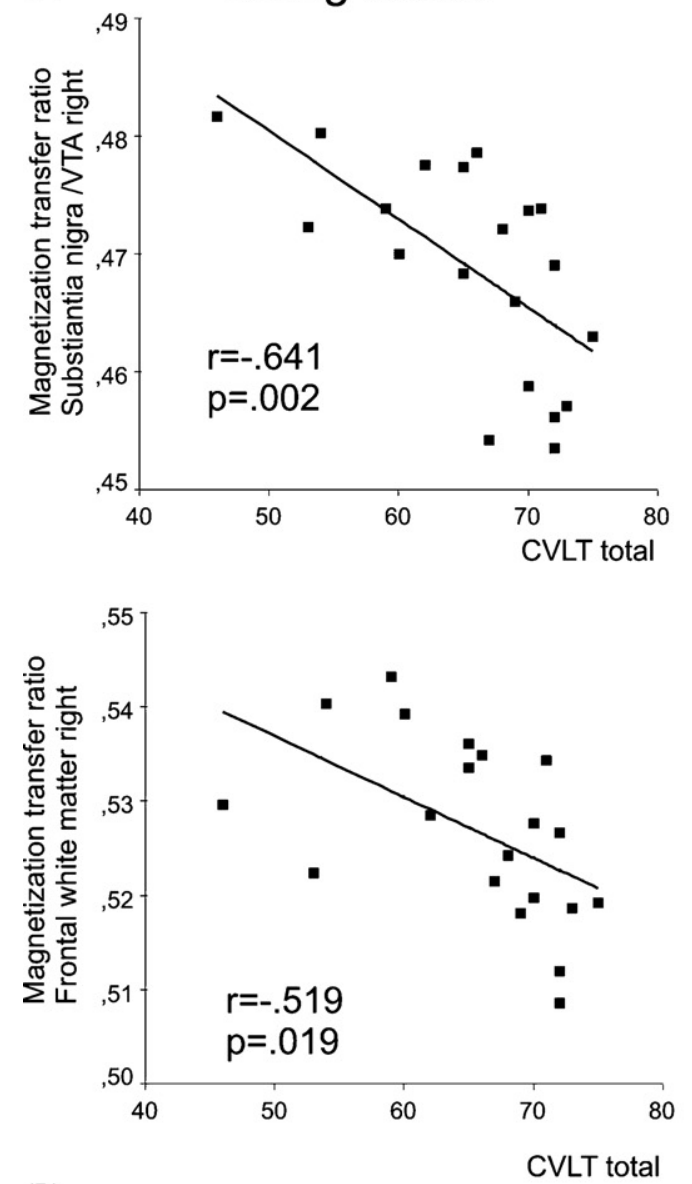

(B)

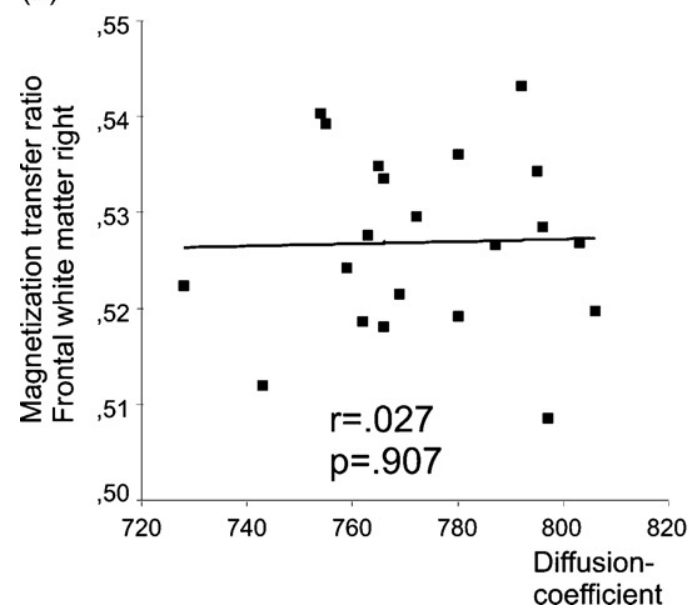

Older adults
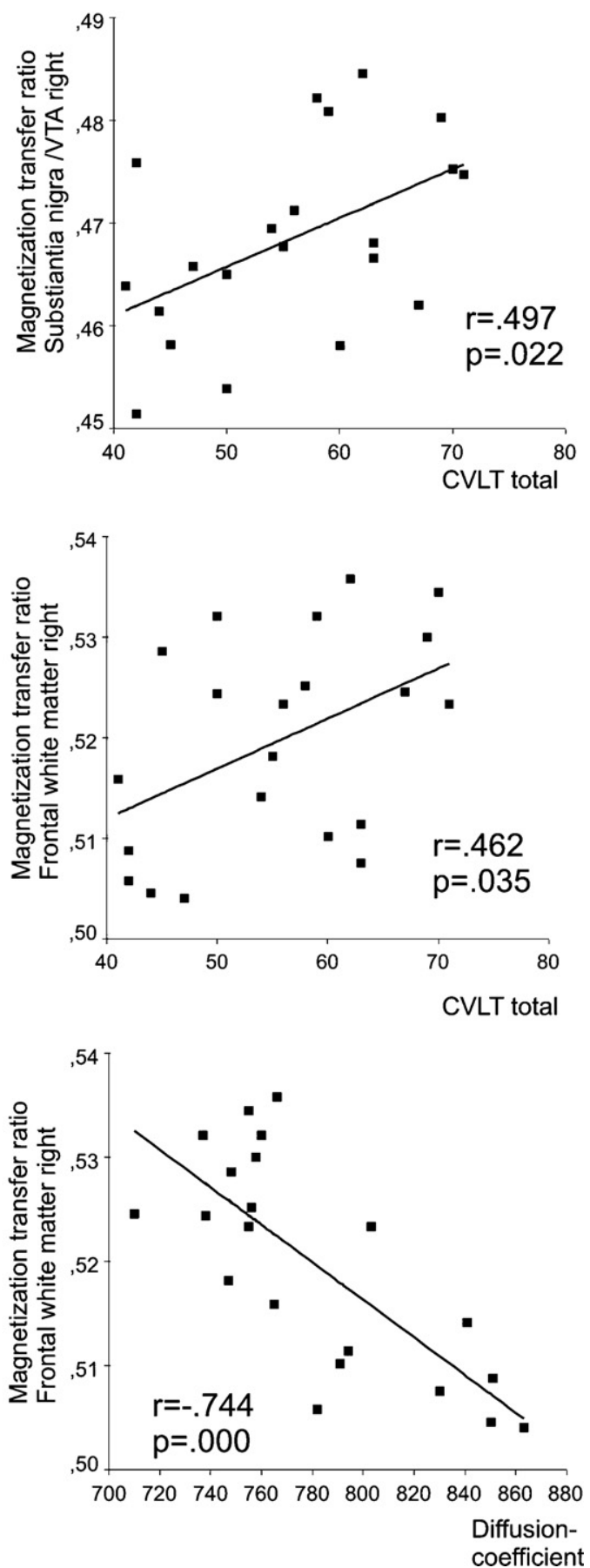

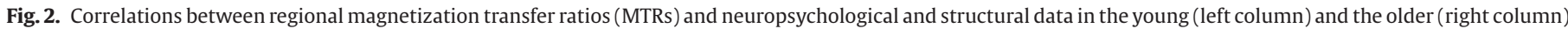

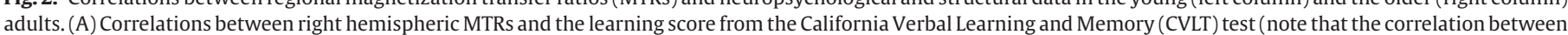

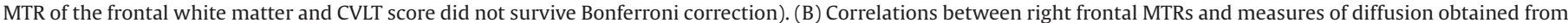
the same region.

between MTR and FWM ADC was not accompanied by an overall decrease of FWM ADC in the older adults compared to the young (Table 1), while FWM AI did show a decrease in the old (Table 1).

Strong differences between the old and the young adults in both ADC and AI emerged in the SN/VTA (Table 1). However, while AI showed the expected decrease in the old, ADC showed a decrease as well (instead of an expected increase in ADC, Table 3) suggesting an overall decrease of SN/VTA water content in aging.

\subsubsection{Multiple regression analyses}

We then used multiple regression analyses to identify how much variance of observed cognitive performance was explained by single or combined regional MTR values and age. Dependent (or predicted) variables were either DS forward, CVLT total or D2-test performance and the four independent (or predictor) variables in each model were MTRs from SN/VTA, hippocampus, FWM and age using the backwards method (note that the 
Table 3

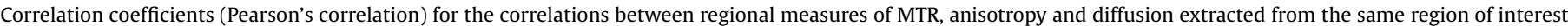
(all from the right hemisphere) for young (upper half) and older (lower half) adults

\begin{tabular}{|c|c|c|c|c|c|c|}
\hline & \multicolumn{6}{|l|}{ MTR } \\
\hline & \multicolumn{2}{|c|}{ Substantia nigra/VTA } & \multicolumn{2}{|c|}{ Hippocampus } & \multicolumn{2}{|c|}{ Frontal white matter } \\
\hline & $r$ & $p$-Value & $r$ & $p$-Value & $r$ & $p$-Value \\
\hline Anisotropy & .236 & .304 & .087 & .872 & .065 & .778 \\
\hline Diffusion & .115 & .620 & .027 & .971 & .172 & .456 \\
\hline Anisotropy & -.105 & .651 & .320 & .154 & .242 & .292 \\
\hline Diffusion & -.146 & .527 & -.337 & .135 & $-.744^{*}$ & .000 \\
\hline
\end{tabular}

The second column of each correlation indicates the statistical significance ( ${ }^{*}$, see text for procedures correcting for multiple comparisons).

ratio between number of subjects and predictor variables was $>5: 1$ ).

No significant regression-model emerged for D2-test as dependent variable for either the young or the older adults $(p>.1)$. For DS forward as independent variable also no significant model emerged in the young, but two significant models emerged in the older adults. The most significant $(F(2,18)=5.185, p=0.017)$ included the predictors age and hippocampus MTR explaining about $37 \%$ of DS forward variance (adjusted $R$ square $=.366$, standardized Betas age $=-.401$, hippocampus MTR $=.384$ ). The second model $(F(3,17)=3.72, p=0.032)$ additionally included the SN/VTA MTR but explained only an additional 3\% of DS forward variance (adjusted $R$ square $=.396$, standardized Betas age $=-.42$, hippocampus MTR=.295, SN/VTA MTR=.196).

Using CVLT total as independent variable three significant models emerged for the young adults. The first $(F(1,19)=12.4, p=0.001)$ included only the predictor SN/VTA MTR and explained about $41 \%$ of CVLT total variance (adjusted $R$ square $=.41$, standardized Beta MTR SN/VTA right $=-.641)$. The other two models, although being significant, explained less than $1 \%$ of additional CVLT variance. The second model $(F(2,18)=6.01, p=0.011)$ additionally included the hippocampus MTR and explained an additional $0.4 \%$ of CVLT total variance (adjusted $R$ square $=.414$, standardized Betas MTR SN/VTA right $=-.575$, hippocampus MTR $=-.089$ ). The third model $(F(3,17)=3.83, p=0.035)$ included the SN/VTA MTR, hippocampus MTR and additionally FWM MTR explaining an additional $0.4 \%$ of CVLT total variance (adjusted $R$ square $=.418$, standardized Betas SN/VTA MTR $=-.471$, hippocampus MTR $=-.120$, FWM MTR = -.106).

Two significant models emerged for the older adults when using CVLT as independent variable. Like in the group of younger adults the first $(F(1,19)=6.239, p=0.022)$ included only one predictor variable, namely, the SN/VTA MTR and explained 25\% of CVLT total variance (adjusted $R$ square $=.247$, standardized Betas MTR SN/VTA right $=.497$ ). Importantly, as already revealed by the correlation analyses, the direction of relationship between CVLT and SN/VTA MTR is positive in the group of older adults and negative in the group of younger adults. The second model $(F(2,18)=4.069$, $p=0.035$ ) also included the FWM MTR and explained an additional $6 \%$ of CVLT total variance (adjusted $R$ square $=.311$, standardized Betas SN/VTA MTR=.357, FWM MTR=.290).

Finally, we determined whether MTR and diffusion based imaging modalities were additive in the amount of CVLT variance they could explain by entering MTR and ADC values into the same model using the backwards model. Neither in the SN/VTA nor in the FWM were models including both modalities significant ( $p s>.1$ ).

\subsubsection{Generalizability of our results}

We finally analyzed to what extent age and neuropsychological and structural parameters of our 21 healthy older adults were a good representation of our entire cohort of 86 healthy adults. Note that by matching for BMI to that of the 21 young adults, we have biased our selection towards a healthier sample than typical of the cohort of 86 adults. To assess generalizabitliy, we expressed the difference between the mean value of each parameter in our selected sample and the parent cohort as a proportion of the standard deviation of that parameter in the parent cohort according to Lindenberger, Singer, \& Baltes (2002):

\section{(Mean sample - Mean parent cohort)) S.D. parent cohort}

By convention (e.g., Cohen, 1977), effect sizes of 0.20, 0.50, and 0.80 are the minimum values required to indicate small, medium, and large effects, respectively. With respect to age, the average magnitude of the selectivity of our sample (0.10S.D. units) was too small to qualify for even a small difference. Of the neuropsychological parameters only CVLT Total showed a small difference (0.3).

The results for all parameters were: Age, 0.1; BMI, 0.56; CVLT Total, 0.3; D2, 0.1; Digit Span, 0.03; MTR SN/VTA right, 0.44; AI SN/VTA right, 0.03; ADC SN/VTA right, 0.52; MTR Hippocampus right, 0.13; AI Hippocampus right, 0.04; ADC Hippocampus right, 0,19; MTR FMW, 0.07; AI FMW, 0.32; ADC FMW, 0.17.

Hence, with respect to age and neuropsychological profile, our sample of 21 healthy older adults was quite typical of our entire cohort, while in terms of BMI and certain structural parameters (see above) they were healthier.

\section{Discussion}

We observed the hypothesized positive correlation between SN/VTA integrity and CVLT performance in the older adults (Fig. 2 Table 2). There was a tendency for such a positive correlation also for the FWM but this did not survive Bonferroni correction (Fig. 2, Table 2) while no correlation emerged for the hippocampus (Table 2). The SN/VTA integrity was not correlated with working memory span (digit span) or attention (D2-test, Table 2), indicating that CVLT was particularly sensitive to SN/VTA integrity. This was confirmed by multiple regression analyses. In both older and younger adults, SN/VTA integrity explained more variance of CVLT total performance than integrity of either the hippocampus or FWM but this was not the case for working memory span and attention.

The finding that SN/VTA integrity impacts on CVLT performance supports our hypothesis that dopaminergic neuromodulation arising from the SN/VTA plays an important role in declarative memory. Dopamine also modulates prefrontal and medial temporal processing, as well as the interaction between medial temporal and prefrontal processing (Grace et al., 2007). To the extent that reductions in SN/VTA MTR are associated with diminished dopaminergic neurotransmission, this may be associated with triple impact upon CVLT: First, there may be a reduction of MTL responses to novelty. We have recently reported (Bunzeck et al., 2007) that reductions in SN/VTA MTR are associated with reductions in hemodynamic novelty responses in medial temporal lobes. As the learning list in CVLT 
is initially novel, reduced novelty responses in the MTL may cause a slowing in learning rate (Tulving et al., 1996). Secondly, reductions in SN/VTA MTR may also negatively impact upon prefrontal mechanisms of cognitive control which are important to be able to effectively control list learning through semantic organization. Third, and finally, these MTL and prefrontal mechanisms need to interface effectively, and reductions in SN/VTA may impair this by disrupting the regulation of information flow between MTL and prefrontal cortex (Grace et al., 2007).

Multiple regression analyses showed that SN/VTA MTR explained more variance of CVLT performance in both young and older adults than MTR of FWM or hippocampus and also more variance than measures of apparent diffusion coefficient. Although this close functional relationship between SN/VTA integrity and CVLT performance was hypothesized it should be noted, that working memory span also seems to be influenced by integrity of the SN/VTA, hippocampus and frontal WM (as apparent from our multiple regression analyses). This is compatible with data indicating that dopaminergic neuromodulation is also related to memory span (Aalto, Bruck, Laine, Nagren, \& Rinne, 2005; Cools, Sheridan, Jacobs, \& D'Esposito, 2007). In our data, memory span was most strongly predicted by age and hippocampal MTR, and adding SN/VTA integrity explained 3\% additional variance of working memory span performance.

\subsection{Structural differences in the SN/VTA of the young and older adults}

We have previously reported MTR reduction in the SN/VTA of our older adults in a different, non-overlapping sample of our population (Bunzeck et al., 2007). Such a reduction is compatible with postmortem evidence clearly demonstrating dopaminergic cell loss in the SN/VTA of healthy old adults (Fearnley \& Lees, 1991b). However, in our present study, we have not observed a significant reduction in the older adults. A key difference between our current study and the previous report is that we have now matched young and old participants in terms of their body mass index (BMI). Indeed, when we chose a different group of older adults that have a higher BMI, we observed a significant reduction in SN/VTA MTR compared to the group of young adults and also compared to a group of older adults with a low BMI.

The availability of diffusion-based SN/VTA measures in addition to the MTR measures allowed us to rule out a number of possible interpretations as to why SN/VTA MTR decreased in adults with high BMI. We observed strong differences between the old and the young adults in ADC and AI of the SN/VTA (Table 1). However, while AI showed the expected decrease in the old, ADC showed a decrease as well (Table 1). Given that ADC is sensitive to capillary expansion, swelling of perivascular spaces and vascular insults (Pfefferbaum, Adalsteinsson, \& Sullivan, 2003; Raz \& Rodrigue, 2006; Wozniak \& Lim, 2006) its decrease in the SN/VTA of older adults strongly argues against the possibility that vascular pathology underlies the normal age-related changes in SN/VTA structure. If that were the case, we would also have expected a correlation between MTR and ADC in the SN/VTA. The diffusion-independent reduction of SN/VTA MTR in high BMI individuals is thus more likely to reflect cellular changes such as a degradation of the neuromelanin macromolecule scaffolding in dopaminergic neurons (Fasano et al., 2006; Seppi \& Schocke, 2005) or trans-synaptic morphological abnormality secondary to afferent demyelinating lesions (Rademacher, Engelbrecht, Burgel, Freund, \& Zilles, 1999; van Waesberghe et al., 1999; Fazekas et al., 2005; Audoin et al., 2006). The decrease in ADC, on the other hand, suggests an overall decrease of SN/VTA water content in aging, the significance of which remains open.

\subsection{Reversed correlations in the young}

We observed a reversed correlation between MTR and CVLT total in the young and older adults. FWM and SN/VTA MTRs correlated negatively with CVLT total in the young, while there was a positive relationship (for the SN/VTA) in the older adults (Fig. 2). Negative correlations between structural measures and cognition in young adults have been reported before (e.g. Schumann et al., 2007; see Van Petten, 2004 for a review based on hippocampal volumes) and suggest that two opposing trends operate in the young and the older adults (e.g. Van Petten, 2004).

In our data set, the availability of diffusion-based measures in addition to the MTR measures allows us to better constrain the interpretation of these opposing trends. In older adults, FWM MTR was likely to be strongly determined by vascular pathology because it was negatively correlated with diffusion (ADC, Fig. 2, Table 3). In the young, on the other hand, we did not observe a significant correlation between MTR, ADC and/or AI. Hence, vascular factors cannot fully explain the relationship between MTR and CVLT in the young. However, there are a number of maturational brain changes that continue into late adolescence (for a review see Paus, 2005), including proliferation of myelin into the peripheral cortical neuropil (Aine et al., 2006; Sowell et al., 2004; Yakovlev \& Lecours, 1967), the formation and usage-dependent selective elimination of synapses (Hensch, 2004; Huttenlocher \& Dabholkar, 1997), and metabolic changes (Chugani, Phelps, \& Mazziotta, 1987), which could contribute to a reversed relationship between MTR and cognition in the young adults. Indeed, we observed that there was an age-related increase in MTRs of both regions within the group of the young adults (Fig. 3) (compatible with previous reports that MTR does not always decrease with age, Armstrong et al., 2004) and that the correlation between MTR and CVLT was weakened when including age as a covariate in this group. This age-dependency supports the notion of maturational factors contributing to the reversed correlation between MTR and CVLT in the young. Among these maturational factors, a strong relationship to myelination is unlikely, as one would also expect this to be accompanied by correlated ADC and AI changes in the young adults (for reviews, see Bartzokis, 2004; Durston et al., 2006; Raz \& Rodrigue, 2006; Wozniak \& Lim, 2006).

\subsection{Hippocampal MTRs}

The older group did not differ in their hippocampus MTR from the younger group which is consistent with data from our recent study in a different sample of old adults (Bunzeck et al., 2007) and also consistent with a number of volumetric studies demonstrating age-related atrophy only in longitudinal but not in cross-sectional studies (e.g. Raz et al., 2005; Szentkuti et al., 2004; for a review, see Van Petten, 2004). Hippocampal reductions of MTR have been reported in Alzheimer's disease (AD) (Hanyu et al., 2000a,b) and to a lesser extent in Lewy body dementia (Hanyu et al., 2005). The specific pathophysiology that underlies the hippocampal MTR reductions in these cases is not yet clear, but our findings of a lack of age-related hippocampal MTR reduction in healthy individuals raise the possibility that hippocampal MTR reductions could be specific to degenerative disorders making it a potentially interesting diagnostic measure.

Although several studies have reported a positive correlation between hippocampal volumes and memory performance in older adults (e.g. Schiltz et al., 2005; Yonelinas et al., 2007; Zimmerman et al., 2008), these findings are unreliable in cross-sectional studies with a number of studies reporting no correlation (for a review, see Van Petten, 2004). Our observation that hippocampal MTR measures did not correlate with CVLT scores is therefore not entirely unexpected. 

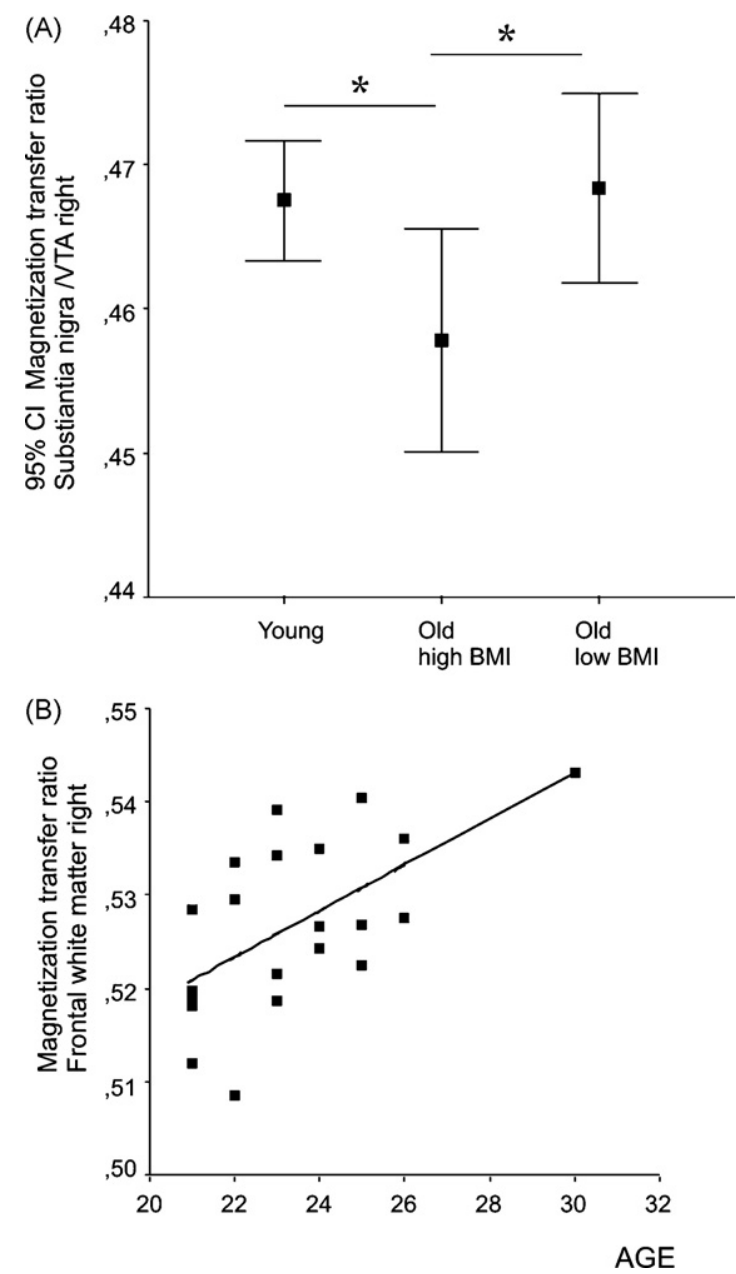

Fig. 3. (A) Comparisons (independent samples $t$-tests) of SN/VTA magnetization transfer ratios (MTRs) between young and older adults, divided into those with high and low body mass index (BMI). (B) Positive correlation between regional magnetization transfer ratio (MTR) of the frontal white matter and age in the group of young adults.

\subsubsection{SN/VTA, mobility, novelty and age-related memory performance}

From an evolutionary perspective, the preferential processing of novel stimuli is regarded as an important mechanism motivating organisms to explore new environments for new sources of reward (Bunzeck \& Duzel, 2006; Kakade \& Dayan, 2002) and it has been suggested that SN/VTA responses to novelty may code this motivational signal that energizes organisms towards exploratory behaviours. In this regard, we see a close relationship between SN/VTA integrity, novelty processing, mobility and memory. We propose that high BMI and low SN/VTA integrity may pose a double burden on memory, by providing less motivational drive to explore in response to novel stimuli, and, at the same time making exploration physically more difficult because of the elevated BMI. As mobility, novelty-exploration and dopaminergic neuromodulation are also linked with hippocampal neurogenesis (Cotman, Berchtold, \& Christie, 2007; Freundlieb et al., 2006; Gould, 2007), the constellation of high BMI and low SN/VTA integrity may have adverse long-term impact on hippocampal plasticity in aging.

In fact, mild motor dysfunction, including impaired gait and balance, is associated with cognitive impairment in aging and is also a predictor of progression into dementia (Boyle et al., 2005; Boyle et al., 2007; Buchman, Boyle, Wilson, Tang, \& Bennett, 2007; Lundin-Olsson, Nyberg, \& Gustafson, 1997). Importantly, vascular risk factors do not account for this relationship (Boyle et al., 2005; Boyle et al., 2007; Buchman et al., 2007; Lundin-Olsson et al., 1997) and it has been suggested that the mild motor impairment may be due to Alzheimer like pathology (e.g. neurofibrillary tangles) in the nigra (Boyle et al., 2005). Evidence is accumulating that even apparently healthy adults may display subtle motor dysfunction associated with mild structural changes in the SN/VTA (e.g. Ross et al., 2004; Ruprecht-Dorfler, Klotz, Becker, \& Berg, 2007) even if, as postmortem studies suggest, they have not developed Parkinson's Disease (Ross et al., 2004). One intriguing possibility therefore is that SN/VTA integrity (in all three dopaminergic pathways) in aging links the motivational and motor aspects of exploratory behaviour and thereby may be a key component underlying the complex relationship between cognition, motivation and mobility with ensuing impact also on BMI. Therefore, understanding the relationship between SN/VTA integrity, BMI, mobility and novelty-processing on memory as well as on the longitudinal trajectory of age-related changes of declarative memory may be a fruitful endeavour in aging-research.

To summarize, our findings suggest that SN/VTA integrity in young and older adults is related to declarative verbal learning and memory performance while showing only a weak relationship to working memory span. Our data highlight the importance of neuromodulatory brain regions in understanding the cognitive profile of age-related memory function. They also raise the possibility that MTR is sensitive to late-maturational changes of frontal white matter and SN/VTA function in young adults. An interesting avenue for the future will be to see whether such structural parameters of the integrity of neuromodulatory brain regions in older adults could motivate specific treatment strategies using dopaminergic agents. To that end, multimodal studies combining structural MRI with in vivo imaging of dopaminergic neurotransmission could be helpful. Developing techniques that are readily usable in healthy adults to assess integrity of key structures of dopaminergic modulation is important. It is evident that MRI-based measures of SN/VTA integrity provide only an indirect link to dopaminergic neuromodulation. However, compared to more direct methods such a positron-emission tomography (e.g. Schott et al., in press), MRIbased methods have the advantage of being repeatable without hazard to health and are therefore important cornerstones of longitudinal studies or studies monitoring the effects of various forms of treatment through repeated scanning.

\section{Acknowledgements}

This study was supported by grants from the Deutsche Forschungsgemeinschaft (Klinische Forschergruppe 'Kognitive Kontrolle von Gedächtnis 163', TP1 \& TP2) and the BMBF (CAI $01 \mathrm{GO0202}$ and 01G00504).

\section{References}

Aalto, S., Bruck, A., Laine, M., Nagren, K., \& Rinne, J. O. (2005). Frontal and temporal dopamine release during working memory and attention tasks in healthy humans: A positron emission tomography study using the high-affinity dopamine D2 receptor ligand [11C]FLB 457. Journal of Neuroscience, 25(10), 2471-2477.

Adcock, R. A., Thangavel, A., Whitfield-Gabrieli, S., Knutson, B., \& Gabrieli, J. D. (2006). Reward-motivated learning: Mesolimbic activation precedes memory formation. Neuron, 50, 507-517.

Aine, C. J., Woodruff, C. C., Knoefel, J. E., Adair, J. C., Hudson, D., Qualls, C., et al. (2006). Aging: Compensation or maturation? Neuroimage, 32, 1891-1904.

Armstrong, C. L., Traipe, E., Hunter, J. V., Haselgrove, J. C., Ledakis, G. E., Tallent, E. M. et al. (2004). Age-related, regional, hemispheric, and medial-lateral differences in myelin integrity in vivo in the normal adult brain. AJNR American Journal of Neuroradiology, 25(6), 977-984.

Audoin, B., Fernando, K. T., Swanton, J. K., Thompson, A. J., Plant, G. T., \& Miller, D. H. (2006). Selective magnetization transfer ratio decrease in the visual cortex following optic neuritis. Brain, 129, 1031-1039. 
Backman, L., Ginovart, N., Dixon, A. R., Wahlin, T. B. R., Wahlin, S. A., Halldin, C., et al. (2000). Age-related cognitive deficits mediated by changes in the striatal dopamine system. American Journal of Psychiatry, 157, 635-637.

Backman, L., Nyberg, L., Lindenberger, U., Li, S. C., \& Farde, L. (2006). The correlative triad among aging, dopamine, and cognition: Current status and future prospects. Neuroscience and Biobehavioral Review, 30, 791-807.

Baddeley, A., Cocchini, G., Della Sala, S., Logie, R. H., \& Spinnler, H. (1999). Working memory and vigilance: Evidence from normal aging and Alzheimer's disease. Brain Cognition, 41, 87-108.

Balota, A. D., Dolan, P. O., \& Duchek, J. M. (2000). Memory changes in healthy older adults. In E. T. a. F. I. M. Craik (Ed.), The Oxford handbook of memory. Oxford: Oxford University Press.

Bartzokis, G. (2004). Age-related myelin breakdown: A developmental model of cognitive decline and Alzheimer's disease. Neurobiology of Aging, 25, 49-62 [5-18 author reply].

Bodammer, N., Kaufmann, J., Kanowski, M., \& Tempelmann, C. (2004). Eddy current correction in diffusion-weighted imaging using pairs of images acquired with opposite diffusion gradient polarity. Magnetic Resonance in Medicine, 51 188-193.

Boyle, P. A., Wilson, R. S., Aggarwal, N. T., Arvanitakis, Z., Kelly, J., Bienias, J. L., et al. (2005). Parkinsonian signs in subjects with mild cognitive impairment. Neurology, 65(12), 1901-1906

Boyle, P. A., Wilson, R. S., Buchman, A. S., Aggarwal, N. T., Tang, Y., Arvanitakis, Z. et al. (2007). Lower extremity motor function and disability in mild cognitive impairment. Experimental Aging Research, 33(3), 355-371.

Brickman, A. M., Habeck, C., Zarahn, E., Flynn, J., \& Stern, Y. (2007). Structural MRI covariance patterns associated with normal aging and neuropsychological functioning. Neurobiology of Aging, 28, 284-295.

Buchman, A. S., Boyle, P. A., Wilson, R. S., Tang, Y., \& Bennett, D. A. (2007). Frailty is associated with incident Alzheimer's disease and cognitive decline in the elderly. Psychosomatic Medicine, 69(5), 483-489.

Buckner, R. L. (2004). Memory and executive function in aging and AD: Multiple factors that cause decline and reserve factors that compensate. Neuron, 44 195-208.

Bunzeck, N., \& Duzel, E. (2006). Absolute coding of stimulus novelty in the human substantia nigra/VTA. Neuron, 51, 369-379.

Bunzeck, N., Schutze, H., Stallforth, S., Kaufmann, J., Duzel, S., Heinze, H. J., et al (2007). Mesolimbic novelty processing in older adults. Cerebral Cortex.

Chugani, H. T., Phelps, M. E., \& Mazziotta, J. C. (1987). Positron emission tomography study of human brain functional development. Annals of Neurology, 22, 487-497.

Cohen, J. (1977). Statistical power analysis for the behavioral sciences (Rev. ed.). New York: Academic Press.

Cools, R., Sheridan, M., Jacobs, E., \& D’Esposito, M. (2007). Impulsive personality predicts dopamine-dependent changes in frontostriatal activity during component processes of working memory. Journal of Neuroscience, 27, 5506-5514.

Cortes, R., Gueye, B., Pazos, A., Probst, A., \& Palacios, J. M. (1989). Dopamine receptors in human brain: Autoradiographic distribution of D1 sites. Neuroscience, 28 , 263-273.

Cotman, C. W., Berchtold, N. C., \& Christie, L. A. (2007). Exercise builds brain health: Key roles of growth factor cascades and inflammation. Trends in Neuroscience $30(9), 464-472$

Craik, F. I. (2006). Brain-behavior relations across the lifespan: A commentary. Neuroscience and Biobehavioral Review, 30, 885-892.

Craik, F. I. M. (1994). Memory changes in normal aging. Current Directions in Psychological Science, 3, 155-158.

Crook, T., Bartus, R., Ferris, S., Whitehouse, P., Cohen, G., \& Gershon, S. (1986). Ageassociated memory impairment: Proposed diagnostic criteria and measures of clinical change-report of a National Institute of Mental Health Work Group Developmental Neuropsychology, 261-276.

Delis, D., Kramer, J., Kaplan, E., \& Ober, B. (1987). California Verbal Learning Test: Adult version. San Antonio, TX: The Psychological Corporation.

Durston, S., Davidson, M. C., Tottenham, N., Galvan, A., Spicer, J., Fossella, J. A., et al. (2006). A shift from diffuse to focal cortical activity with development. Developmental Science, 9, 1-8.

Eckert, T., Sailer, M., Kaufmann, J., Schrader, C., Peschel, T., Bodammer, N., et al. (2004). Differentiation of idiopathic Parkinson's disease, multiple system atrophy, progressive supranuclear palsy, and healthy controls using magnetization transfer imaging. Neuroimage, 21, 229-235.

Fasano, M., Bergamasco, B., \& Lopiano, L. (2006). Modifications of the ironneuromelanin system in Parkinson's disease. Journal of Neurochemistry, 96 909-916.

Fearnley, J. M., \& Lees, A. J. (1991a). Ageing and Parkinson's disease: Substantia nigra regional selectivity. Brain, 114(Pt. 5), 2283-2301.

Fearnley, J. M., \& Lees, A. J. (1991b). Ageing and Parkinson's disease: Substantia nigra regional selectivity. Brain, 2283-2301.

Freundlieb, N., Francois, C., Tande, D., Oertel, W. H., Hirsch, E. C., \& Hoglinger, G. U. (2006). Dopaminergic substantia nigra neurons project topographically organized to the subventricular zone and stimulate precursor cell proliferation in aged primates. Journal of Neuroscience, 26(8), 2321-2325.

Good, C. D., Johnsrude, I. S., Ashburner, J., Henson, R. N., Friston, K. J., \& Frackowiak, R. S. (2001). A voxel-based morphometric study of ageing in 465 normal adult human brains. Neuroimage, 14, 21-36.

Gould, E. (2007). How widespread is adult neurogenesis in mammals? Nature Reviews. Neuroscience, 8(6), 481-488.
Grace, A. A., Floresco, S. B., Goto, Y., \& Lodge, D. J. (2007). Regulation of firing of dopaminergic neurons and control of goal-directed behaviors. Trends in Neuroscience, 30, 220-227.

Grady, C. L., \& Craik, F. I. (2000). Changes in memory processing with age. Current Opinion in Neurobiology, 10, 224-231.

Hanyu, H., Asano, T., Iwamoto, T., Takasaki, M., Shindo, H., \& Abe, K. (2000). Magnetization transfer measurements of the hippocampus in patients with Alzheimer's disease, vascular dementia, and other types of dementia. AJNR American Journal of Neuroradiology, 21, 1235-1242.

Hanyu, H., Asano, T., Kogure, D., Sakurai, H., Iwamoto, T., \& Takasaki, M. (2000). Relation between hippocampal damage and cerebral cortical function in Alzheimer's disease. Nippon Ronen Igakkai Zasshi, 37, 921-927.

Hanyu, H., Shimizu, S., Tanaka, Y., Kanetaka, H., Iwamoto, T., \& Abe, K. (2005). Differences in magnetization transfer ratios of the hippocampus between dementia with Lewy bodies and Alzheimer's disease. Neuroscience Letters, 380, 166-169.

Hedden, T., \& Gabrieli, J. D. (2004). Insights into the ageing mind: A view from cognitive neuroscience. Nature Reviews. Neuroscience, 5, 87-96.

Hensch, T. K. (2004). Critical period regulation. Annual Reviews of Neuroscience, 27. 549-579.

Huttenlocher, P. R., \& Dabholkar, A. S. (1997). Regional differences in synaptogenesis in human cerebral cortex. The Journal of Comparative Neurology, 387, 167-178.

Kakade, S., \& Dayan, P. (2002). Dopamine: Generalization and bonuses. Neural Networks, 15, 549-559.

Kray, J., \& Lindenberger, U. (2000). Adult age differences in task switching. Psychology and Aging, 15, 126-147.

Lehrl, S. (2005). Mehrfachwahl-Wortschatz-Intelligenztest MWT-B. 5. Aufl. Balingen: Spitta Verlag.

Lindenberger, U., Marsiske, M., \& Baltes, P. B. (2000). Memorizing while walking: Increase in dual-task costs from young adulthood to old age. Psychology and Aging, 15, 417-436.

Lindenberger, U., Singer, T., \& Baltes, P. B. (2002). Longitudinal selectivity in aging populations: Separating mortality-associated versus experimental components in the Berlin Aging Study (BASE). Journal of Gerontology: Psychological Sciences, $6,474-482$.

Lisman, J. E., \& Grace, A. A. (2005). The Hippocampal-VTA Loop: Controlling the entry of information into long-term memory. Neuron, 46, 703-713.

Lisman, J. E., \& Otmakhova, N. A. (2001). Storage, recall, and novelty detection of sequences by the hippocampus: Elaborating on the SOCRATIC model to account for normal and aberrant effects of dopamine. Hippocampus, 11, 551-568.

Lundin-Olsson, L., Nyberg, L., \& Gustafson, Y. (1997). "Stops walking when talking" as a predictor of falls in elderly people. Lancet, 349(9052), 617.

Mishkin, M., Suzuki, W. A., Gadian, D. G., \& Vargha-Khadem, F. (1997). Hierarchical organization of cognitive memory. Philosophical Transactions of the Royal Society of London B. Biological Science, 352, 1461-1467.

Moseley, M. (2002). Diffusion tensor imaging and aging-A review. NMR Biomedicine, $15,553-560$.

Mungas, D., Harvey, D., Reed, B. R., Jagust, W. J., DeCarli, C., Beckett, L., et al. (2005). Longitudinal volumetric MRI change and rate of cognitive decline. Neurology, 65, 565-571.

Nilsson, L. G. (2003). Memory function in normal aging. Acta Neurologica Scandinavica Supplementum, 179, 7-13

Papadakis, N. G., Xing, D., Houston, G. C., Smith, J. M., Smith, M. I., James, M. F., et al. (1999). A study of rotationally invariant and symmetric indices of diffusion anisotropy. Magnetic Resonance Imaging, 17, 881-892.

Parkin, A. J., \& Java, R. I. (1999). Deterioration of frontal lobe function in normal aging: Influences of fluid intelligence versus perceptual speed. Neuropsychology, $13,539-545$

Paus, T. (2005). Mapping brain maturation and cognitive development during adolescence. Trends in Cognition Science, 9, 60-68.

Pfefferbaum, A., Adalsteinsson, E., \& Sullivan, E. V. (2003). Replicability of diffusion tensor imaging measurements of fractional anisotropy and trace in brain.Journal of Magnetic Resonance Imaging, 18, 427-433.

Rademacher, J., Engelbrecht, V., Burgel, U., Freund, H., \& Zilles, K. (1999). Measuring in vivo myelination of human white matter fiber tracts with magnetization transfer MR. Neuroimage, 9, 393-406.

Raz, N., Lindenberger, U., Rodrigue, K. M., Kennedy, K. M., Head, D., Williamson, A. et al. (2005). Regional brain changes in aging healthy adults: General trends, individual differences and modifiers. Cerebral Cortex, 15(11), 1676-1689.

Raz, N., \& Rodrigue, K. M. (2006). Differential aging of the brain: Patterns, cognitive correlates and modifiers. Neuroscience and Biobehavioral Reviews, 30, 730-748.

Reitan, R. M. (1992). The trail making test: Manual for administration and scoring. Tucson: Neuropsychology Laboratory.

Rinne, J. O., Lonnberg, P., \& Marjamaki, P. (1990). Age-dependent decline in human brain dopamine D1 and D2 receptors. Brain Research, 508, 349-352.

Ross, G. W., Petrovitch, H., Abbott, R. D., Nelson, J., Markesbery, W., Davis, D., et al. (2004). Parkinsonian signs and substantia nigra neuron density in decendents elders without PD. Annals of Neurology, 56(4), 532-539.

Ruff, R. M., Light, R. H., Parker, S. B., \& Levin, H. S. (1997). The psychological construct of word fluency. Brain Language, 57, 394-405.

Ruprecht-Dorfler, P., Klotz, P., Becker, G., \& Berg, D. (2007). Substantia nigra hyperechogenicity correlates with subtle motor dysfunction in tap dancers. Parkinsonism \& Related Disorders, 13(6), 362-364.

Salthouse, T. A. (2000). Aging and measures of processing speed. Biological Psychology, 54, 35-54 
Salthouse, T. A. (2003). Memory aging from 18 to 80. Alzheimer Disease and Associated Disorders, 17, 162-167.

Schiltz, K., Szentkuti, A., Guderian, S., Kaufmann, J., Munte, T. F., Heinze, H. J., et al. (2006). Relationship between hippocampal structure and memory function in elderly humans. Journal of Cognitive Neuroscience, 18, 990-1003.

Schott, B. H., Minuzzi, L., Krebs, R. M., Elmenhorst, D., Lang, M., Winz, O., et al. (in press). Mesolimbic fMRI activations during reward anticipation correlate with reward-related ventral striatal dopamine release, Journal of Neuroscience.

Schott, B. H., Seidenbecher, C. I., Fenker, D. B., Lauer, C. J., Bunzeck, N., Bernstein, H. G., et al. (2006). The dopaminergic midbrain participates in human episodic memory formation: Evidence from genetic imaging. Journal of Neuroscience, 26, 1407-1417.

Schumann, C. M., Hamstra, J., Goodlin-Jones, B. L., Kwon, H., Reiss, A. L., \& Amaral, D. G. (2007). Hippocampal size positively correlates with verbal IQ in male children. Hippocampus, 17, 486-493.

Seeman, P., Bzowej, N. H., Guan, H. C., Bergeron, C., Becker, L. E., Reynolds, G. P., et al. (1987). Human brain dopamine receptors in children and aging adults. Synapse, 1, 399-404.

Seppi, K., \& Schocke, M. (2005). An update on conventional and advanced magnetic resonance imaging techniques in the differential diagnosis of neurodegenerative parkinsonism. Current Opinion in Neurology, 18, 370-375.

Snow, B. J., Tooyama, I., McGeer, E. G., Yamada, T., Calne, D. B., Takahashi, H., et al. (1993). Human positron emission tomographic [18F]fluorodopa studies correlate with dopamine cell counts and levels. Annals of Neurology, 34, 324-330.

Sowell, E. R., Thompson, P. M., Leonard, C. M., Welcome, S. E., Kan, E., \& Toga, A. W. (2004). Longitudinal mapping of cortical thickness and brain growth in normal children. Journal of Neuroscience, 24, 8223-8231.

Squire, L. R., Stark, C. E., \& Clark, R. E. (2004). The medial temporal lobe. Annual Reviews of Neuroscience, 27, 279-306.

Stuss, D. T., \& Levine, B. (2002). Adult clinical neuropsychology: Lessons from studies of the frontal lobes. Annual Review of Psychology, 53, 401-433.

Szentkuti, A., Guderian, S., Schiltz, K., Kaufmann, J., Munte, T. F., Heinze, H. J., et al. (2004). Quantitative MR analyses of the hippocampus: Unspecific metabolic changes in aging. Journal of Neurology, 251(11), 1345-1353.
Tulving, E. (1985). Memory and consciousness. Canadian Psychology, 26, 1-12.

Tulving, E., Markowitsch, H. J., Craik, F. E., Habib, R., \& Houle, S. (1996). Novelty and familiarity activations in PET studies of memory encoding and retrieval. Cerebral Cortex, 6(1), 71-79.

Van Petten, C. (2004). Relationship between hippocampal volume and memory ability in healthy individuals across the lifespan: Review and meta-analysis Neuropsychologia, 42(10), 1394-1413.

Weidlich, S., \& Lamberti, G. (1993). DCS Diagnosticum für Cerebralschädigungen. Bern, Switzerland: Hans Huber.

WHO, \& ISH. (1999). WHO/ISH-Guidelines-Subcommittee: World Health Organization-International Society of Hypertension Guidelines for the Management of Hypertension. Journal Hypertension, 151-183.

Wittmann, B. C., Schott, B. H., Guderian, S., Frey, J. U., Heinze, H. J., \& Duzel, E. (2005) Reward-related FMRI activation of dopaminergic midbrain is associated with enhanced hippocampus-dependent long-term memory formation. Neuron, 45 , 459-467.

Wolff, S. D., \& Balaban, R. S. (1989). Magnetization transfer contrast (MTC) and tissue water proton relaxation in vivo. Magnetic Resonance in Medicine, 10 135-144.

Woods, R. P., Grafton, S. T., Holmes, C. J., Cherry, S. R., \& Mazziotta, J. C. (1998). Automated image registration. I. General methods and intrasubject, intramodality validation. Journal of Computer Assisted Tomography, 22, 139-152.

Wozniak, J. R., \& Lim, K. O. (2006). Advances in white matter imaging: A review of in vivo magnetic resonance methodologies and their applicability to the study of development and aging. Neuroscience and Biobehavioral Reviews, 30 762-774.

Yakovlev, P. I., \& Lecours, A. R. (1967). Regional development of the brain in early life. Oxford: Blackwell Scientific.

Yonelinas, A. P., Widaman, K., Mungas, D., Reed, B., Weiner, M. W., \& Chui, H. C. (2007). Memory in the aging brain: Doubly dissociating the contribution of the hippocampus and entorhinal cortex. Hippocampus, 17(11), 1134-1140.

Zimmerman, M. E., Pan, J. W., Hetherington, H. P., Katz, M. J., Verghese, J., Buschke, H., et al. (2008). Hippocampal neurochemistry, neuromorphometry, and verbal memory in nondemented older adults. Neurology. 